\title{
Breathing as One of the Components of Biathlon Shooting in Youth Biathletes
}

\author{
Michal Žák, Jan Ondráček \\ Masaryk University, Faculty of Sports Studies, Brno, Czech Republic
}

\begin{abstract}
PURPOSE: This study is aimed at identifying the length of breath holding in youth biathletes before shooting in both the prone and the standing position, and determining potential dependence between breath holding and the shooting performance at rest and after physical load during the training period. METHODS: Twenty-one national youth biathletes, women $(n=10 ; 16.7 \pm 0.7$ years) and men $(n=11$; $16.5 \pm 0.6$ years), participated in the study. All participants completed two series of measuring of breath holding after 3 months period during shooting at rest and after roller skiing in a race load, in the prone as well as the standing position. They shot using their own biathlon rifles with a fixed accelerometer and respiration belts on the thorax.
\end{abstract}

RESULTS: The average length of breath holding when successfully hitting the target in the prone position was 0.65 seconds in average both at rest and in the racing load, with some significant differences both men and women. When shooting in the standing position, the average values were approximately same as in the prone position 0.65 seconds both at rest and in racing load, again with some observable differences both men and women. There were several significant differences between the pre-test and post-test in the length of breath holding in both groups $(p<0.05)$.

CONCLUSIONS: The study evaluate the length of breath holding and determine dependence of breath holding on the shooting performance at rest and after physical load during the training period in youth biathletes. The comparison of the acquired information with the shooting methodology taught by the coaches brought beneficial results. The study showed that is appropriate to hold breath for an average of $0.55-0.7$ seconds in both shooting positions for both men and women in this age category.

Keywords: biathlon training; breath holding; physical load; shooting performance; training period.

\section{INTRODUCTION}

Biathlon is a sport that connects two seemingly different disciplines: cross-country skiing and shooting. Both disciplines have their specifics. Cross-country skiing makes high demands on the cardio-respiratory system and movement preconditions of the athletes which, along with many other factors, affect the shooting performance. Considering the shooting technique, we define the internal factors of the biathlete and other components of shooting such as the shooting position, postural stability, physical load, aiming, breathing, triggering and many more (Žák et al., 2020). Numerous authors deal with the influence of these factors on the success of shooting. However, a minimal number of studies focuses exclusively on breathing in biathlon shooting.

The respiratory rate (RR) is one of factors that can affect the shooting performance. RR depends mainly on the level of physical activity. Pelin et al. (2020) wrote that anaerobic stress affects both the disciplines, cross-country skiing and shooting, due to increased respiratory activity and heart rate (HR), while also having a negative effect on postural regulation. The authors agreed that physical stress, i.e. high $\mathrm{HR}$ and RR, in biathletes adversely affects the accuracy, shooting time, 
postural control and stability of the rifle, with a greater impact on the standing position than on the prone position (Ihalainen et al., 2018; Laaksonen et al., 2018; Pelin et al., 2020; Sattlecker et al., 2017). Ihalainen et al. (2018) stated that biathletes usually hold their breath during the aiming and triggering phases, which means that the observed decrease in postural balance during intense exercise is likely caused by other mechanisms, such as higher cardiovascular load, than the increased breathing activity. Pelin et al. (2018) mentions the quality of the breathing technique, which is determined by the uniformity of the interval between shots, a balanced rhythm (a continuous succession of inspiration, expiration and apnea) and by appropriate coordination with other elements of the technique. Furthermore, Pelin et al. (2019) focused on adjusting HR before entering the shooting range in young biathletes. They achieved efficiency and progress in shooting from both prone and standing positions. Høydal and Nord (2017) also emphasized the importance of HR monitoring during young biathletes' training as one of the predictors of successful shooting.

Another important factor which is associated with the length of breath holding during shooting is aiming. Vonheim (2012) investigated the effect of dynamic and isometric aiming at rest and after physical load. The resulting claim states that there are various factors which make it difficult to compare the holding still and the following the line approaches. The differences were reported not to be substantial enough to determine without doubt if the subjects perform better utilizing dynamic precision (following the line) rather than the isometric precision (holding still), or if the isometric precision is more affected by the intensity of the physical load than the dynamic precision. Moreover, he found that increased HR appeared to cause more difficulties for the subjects to hold their breath and aim controllably. Therefore, the subjects performed their shooting faster to avoid hypoxia or breathing during aiming (Vonheim, 2012).

Higginson's research into breathing during shooting (2002) was also very beneficial to our study. He stated that RR is substantially elevated upon entering the range during a biathlon race due to the increased physiological demand placed on the human body at high levels of physical activity. The most apparent implication related to a high $\mathrm{RR}$ is the movement of the rifle during inspiration and expiration, resulting in a lower stability of hold. The reasoning behind this phenomenon is that biathletes time their shots according to their breathing pattern, usually taking one breath per shot. Moreover, respiration also plays an indirect role in the stability of hold. Many studies reported by Higginson (2002) have shown that respiration is the primary determinant of HR. These studies indicated that rapid shallow breathing decreases HR and heart rate variability (HRV), whereas slow deep breathing increases HR and HRV.

The above-mentioned articles deal mainly with the body's response to physical activity and examine HR and respiration before and during shooting. However, no research has focused purely on the duration of breath holding during shooting. This is a very individual matter related to local shooting methodologies. The length of breath holding usually correlates with the length of aiming at the target. In the prone position it should be longer than in the standing position. This study aims to discover the specific length of breath holding in young biathletes. Couches in the Czech Republic teach young biathletes to hold their breath for 0.5 to 1 second before triggering in the prone position and for 0.2 to 1 second in the standing position. Our objective was to verify and evaluate this fact by studying the dependence between the length of breath holding and the shooting success. The following research question was asked: RQ1: What is the difference between the length of breath holding at rest and after physical load? RQ2: How will the length of breath holding at rest and after physical activity change after a 3-month intervention? RQ3: What is the difference in the length of breath holding between hit and missed target? 


\section{MATERIAL AND METHODS}

\section{Participants}

Twenty-one national youth biathletes (ten women, eleven men) participated in the study. Participants were members of the Biathlon youth sports center in the Czech Republic. They represented the highest national level in the selected category. The mean and standard deviation of age, height, and weight were $16.7 \pm 0.7$ years, $166.1 \pm 4.9 \mathrm{~cm}, 58.8 \pm 5.7 \mathrm{~kg}$ for women and 16.5 \pm 0.6 years, $177.7 \pm 4.8 \mathrm{~cm}, 67.1 \pm 5.4 \mathrm{~kg}$ for men. The study was conducted in accordance with the Declaration of Helsinki and followed the ethical standards of the Masaryk University. Before the study, all participants were informed about the aim of the study, the nature, and potential risks of the study and that the data will be processed anonymously. Each of the participants (or their legal representative in the case of under-age athletes) signed an informed consent in which they voluntarily agreed to take part in the research.

\section{Test design}

All tests were conducted at the Vysočina Arena in Nové Město na Moravě, Czech Republic (600 $\mathrm{m}$ above sea level), which is a certified outdoor biathlon stadium. Two testing measurements were obtained after 3 months in July 2020 (pre-test) and in October 2020 (post-test), in both cases between 1 p.m. and 4 p.m. The weather conditions were as follows: average temperature $21{ }^{\circ} \mathrm{C}$; sunny / partly cloudy; calm / light air. Participants shot with their own rifles and were instructed to shoot in their individual firing rhythms and speeds. Before the test, they calibrated the rifles in both prone and standing positions (15-20 shots per athlete). The test had the following form (Figure 1). Each participant shot 1 series of 5 shots in both prone and standing positions at rest $\rightarrow$ warmed up for $15 \mathrm{~min}$ at a gradually increasing speed on roller skies $\rightarrow$ completed 3 min ride in race load (RL; $95 \%$ of $\mathrm{HR}_{\max }$ ) $\rightarrow$ shot 5 shots in prone in $\mathrm{RL} \rightarrow$ completed 2 min ride in $\mathrm{RL} \rightarrow$ shot 5 shots in prone in $\mathrm{RL} \rightarrow$ completed 2 min ride in $\mathrm{RL} \rightarrow$ shot 5 shots in standing position in $\mathrm{RL} \rightarrow$ completed 2 min ride in $\mathrm{RL} \rightarrow$ shot 5 shots in standing position in RL. Finally, there was a cooling phase in the form of roller skiing. The researchers monitored the participants' HR during roller skiing and shooting using a Polar sport tester (model Polar M600). The participants fired at metal targets in the test $(4.5 \mathrm{~cm}$ in diameter for the prone position; $11.5 \mathrm{~cm}$ in diameter for the standing position; $50 \mathrm{~m}$ distance). The participants were not provided any feedback about the results during the whole period of the experiment. 


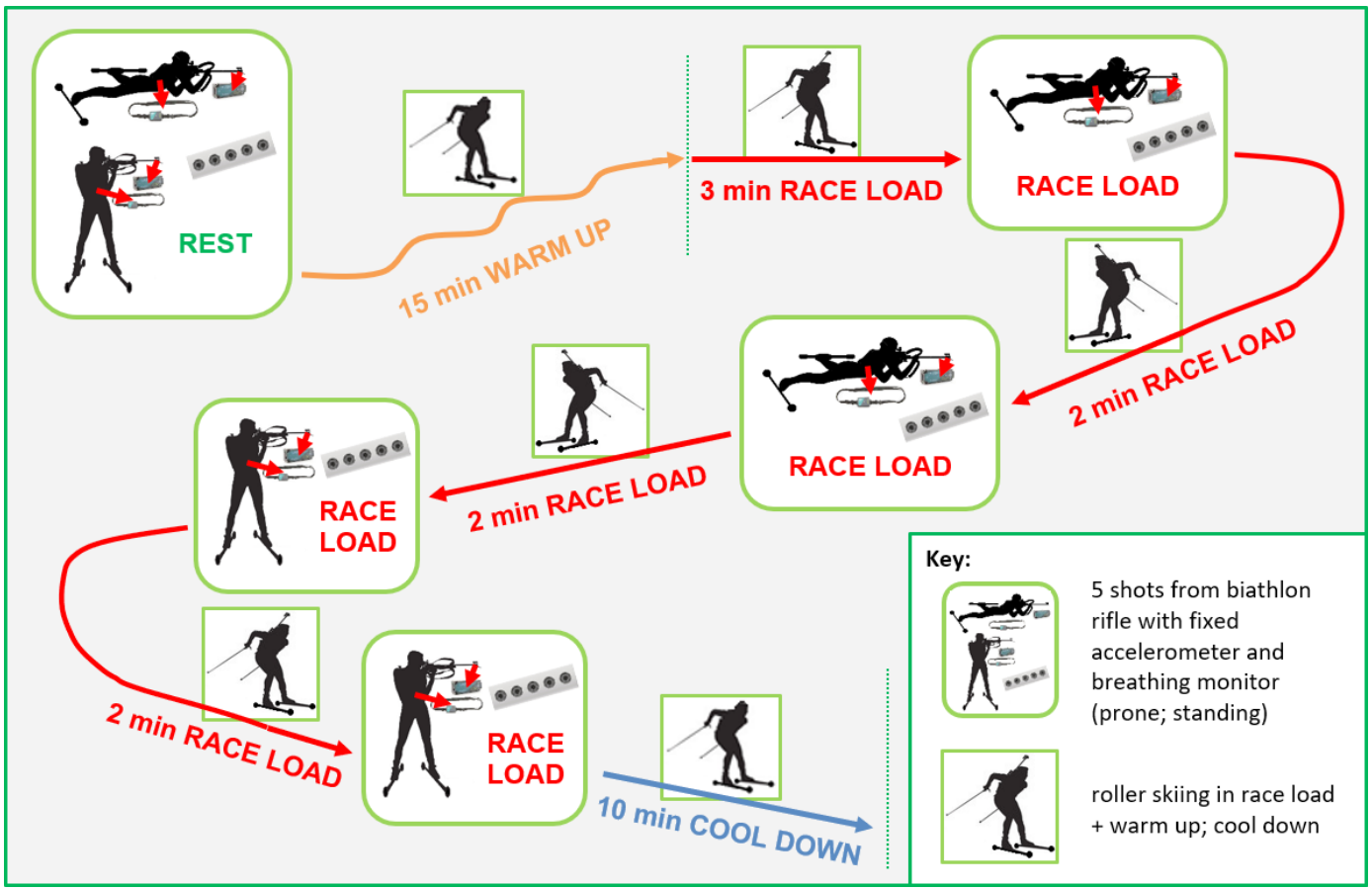

Figure 1. Graphical form of the test design

\section{Measurement of breathing during shooting}

Breathing during shooting was monitored and recorded using a Go Direct ${ }^{\circledast}$ Respiration Belt, Go Direct ${ }^{\oplus}$ Acceleration Sensor and Vernier Graphical Analysis software (Vernier Software \& Technology). The Go Direct ${ }^{\oplus}$ Respiration Belt was placed on the participant's' thorax (in the prone position) or abdomen (in the standing position). The 3-axis accelerometer Go Direct $^{\oplus}$ Acceleration Sensor was fixed (Figure 2) on the top of the barrel of the biathlon rifle (ANSCHÜTZ, model $1827 \mathrm{~F}$ ). The data were sampled at $100 \mathrm{~Hz}$. All information was graphically displayed in the researcher's computer using the Vernier Graphical Analysis software (Figure 3). For evaluating the results, the use of an accelerometer helped to accurately determine the moment of the shot during breathing or holding the breath, respectively. The essential information was the length of breath holding in relation to the success of the shooting. The indication of the moment the shooter stopped breathing required manual marking on the timeline. Subsequently, the time difference between stopping the breathing and shooting was calculated. 


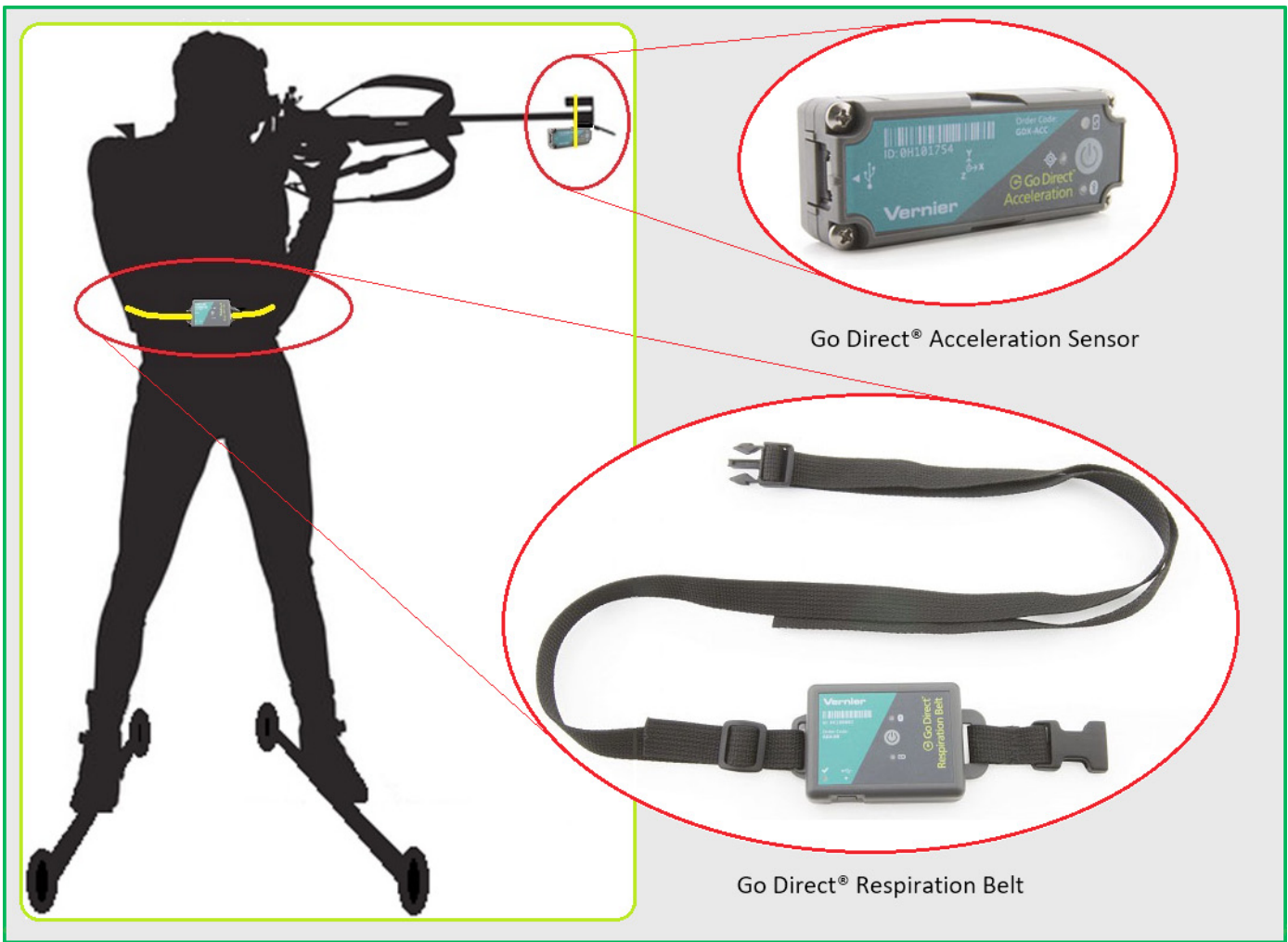

Figure 2. Location of sensors measurement

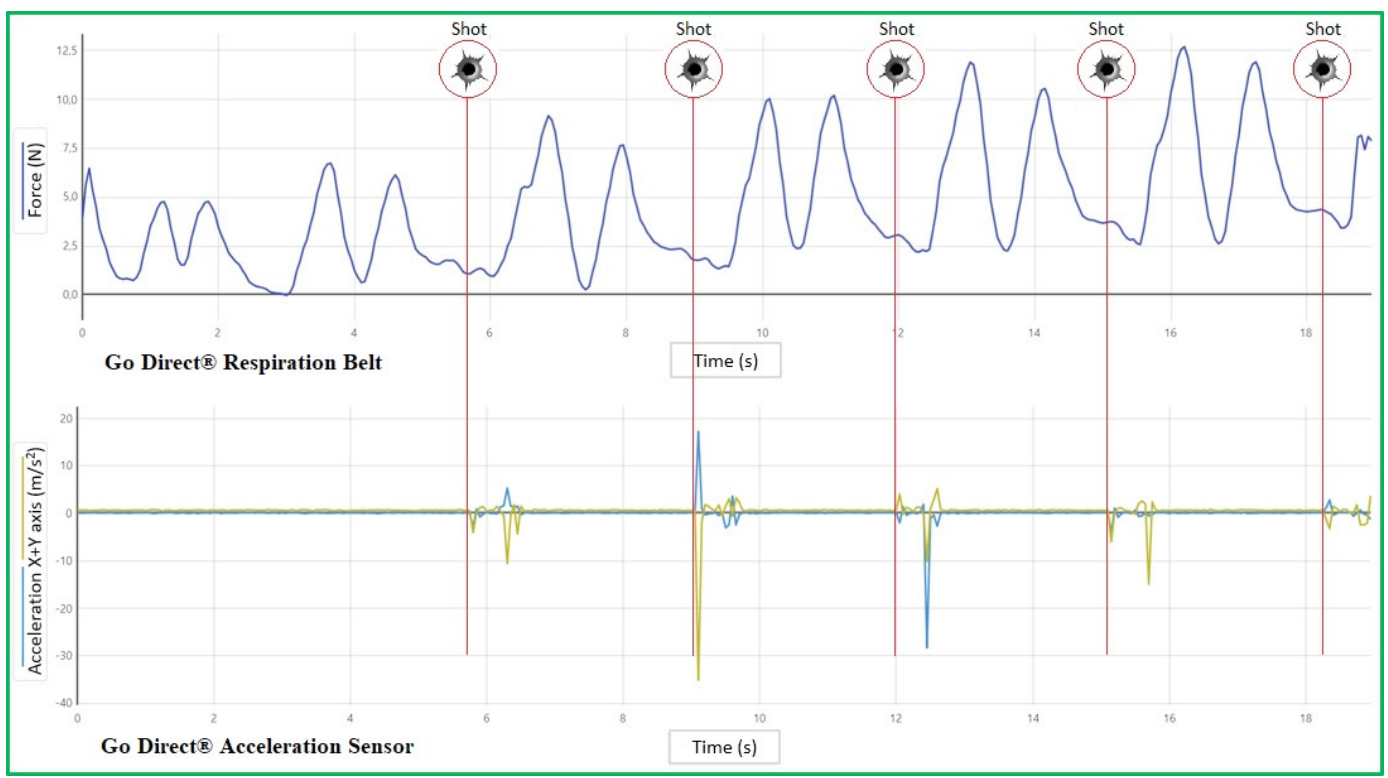

Figure 3. Working with data in Vernier Graphical Analysis software

\section{Statistical analysis}

The statistical analyses were conducted using the STATISTICA software (Statsoft Inc, Tulsa, Oklahoma, USA; version 12). Descriptive data were summarized as mean \pm standard deviation 
(SD). Shapiro-Wilk's test was used to test the normality of the data. For the statistical analysis, unpaired sample $t$-tests were compared in both the men and the women groups for both the prone and the standing positions: breath holding at rest vs. in race load (RL) (pre-test; hit), breath holding at rest vs. in RL (post-test; hit), breath holding at rest in pre-test vs. post-test (hit), breath holding in RL in pre-test vs. post-test (hit), breath holding at rest in hit vs. missed targets in pretests vs. post-tests, breath holding in RL in hit vs. miss ed targets in pre-tests vs. post-tests. The level of significance $(\alpha)$ was set at $p<0.05$. It was also calculated the statistical significance of the effect size (ES) to determine the effect of the differences between given indicators (effect size index Cohen's $d$ ). According to Cohen (1988), the effect size was interpreted as small $(d=0.20)$, medium $(d=0.50)$ or large $(d=0.80)$. Values less than small were marked as trivial.

\section{RESULTS}

All obtained data are presented in Tables 1-6 below. The columns show the values of the length of breath holding (in seconds) for women in the prone position (Women_P) and the standing position (Women_S), as well as for men in the prone position (Men_P) and the standing position (Men_S). The rows indicate individual parameters such as load intensity (REST, racing load $(\mathrm{RL})$ ), measurement period (pre-test, post-test), number of shots fired in given indicators $\left(\mathrm{n}_{\mathrm{s}}\right)$, successfully hit targets (hit) and missed targets (miss). Furthermore, a statistical significance between each two parameters ( $p$-value) and effect size (Cohen's $d$ ) is given. Statistical methods describe equality of mean values of these variables.

If the biathlete is well trained, the apnea pause should be as long at rest and after physical load. The first two tables (Table 1, Table 2) show the differences in the length of breath holding between rest and race load in successfully hit targets in pre-test and post-test. The results answered to RQ1: What is the difference between the length of breath holding at rest and after physical load? No statistically significant dependence was found among most of the monitored indicators. In the case of standing shooting in a pre-test in July in women was statistical significance found $(p<0.05)$, when the women held their breath longer after the race load. Cohen's $d$ calculation showed a medium effect size in this one case $(d=0.53)$, in other cases were values of Cohen's $d$ trivial or small.

Table 1. Comparison of breath holding (second) at REST (pre-test; hit) vs. in RL (pre-test; hit)

\begin{tabular}{l|cc:cc:cc:cc}
\hline & Women_P & $\mathrm{n}_{\mathrm{s}}$ & Men_P & $\mathrm{n}_{\mathrm{s}}$ & Women_S & $\mathrm{n}_{\mathrm{s}}$ & Men_S & $\mathrm{n}_{\mathrm{s}}$ \\
\hline REST_pre-test_hit & $0.53 \pm 0.32$ & 44 & $0.58 \pm 0.36$ & 44 & $0.42 \pm 0.36$ & 42 & $0.61 \pm 0.43$ & 44 \\
RL_pre-test_hit & $0.61 \pm 0.36$ & 68 & $0.75 \pm 0.63$ & 78 & $0.63 \pm 0.43$ & 75 & $0.62 \pm 0.57$ & 81 \\
p-value & $\mathrm{NS}(.190)$ & & $\mathrm{NS}(.099)$ & & ${ }^{*} .009$ & & $\mathrm{NS}(.921)$ \\
Cohen's $\boldsymbol{d}$ & 0.26 & & 0.32 & & ${ }^{* *} 0.53$ & & \multicolumn{2}{c}{0.02} \\
\hline
\end{tabular}

Data are presented as mean $\pm \mathrm{SD}$; NS: not statistically significant; ${ }^{\star} p<.05 ;{ }^{* \star} d \geq .50 ;{ }^{\star *} d \geq .80$

Table 2. Comparison of breath holding (second) at REST (post-test; hit) vs. in RL (post-test; hit)

\begin{tabular}{l|cc:cc:cc:cc}
\hline & Women_P & $\mathrm{n}_{\mathrm{s}}$ & Men_P & $\mathrm{n}_{\mathrm{s}}$ & Women_S & $\mathrm{n}_{\mathrm{s}}$ & Men_S & $\mathrm{n}_{\mathrm{s}}$ \\
\hline REST_post-test_hit & $0.62 \pm 0.31$ & 41 & $0.72 \pm 0.56$ & 46 & $0.66 \pm 0.50$ & 36 & $0.57 \pm 0.46$ & 39 \\
RL_post-test_hit & $0.69 \pm 0.37$ & 68 & $0.59 \pm 0.46$ & 90 & $0.64 \pm 0.45$ & 70 & $0.57 \pm 0.35$ & 57 \\
p-value & $\mathrm{NS}(.356)$ & & $\mathrm{NS}(.136)$ & $\mathrm{NS}(.836)$ & $\mathrm{NS}(.948)$ & \\
Cohen's $\boldsymbol{d}$ & 0.19 & & 0.26 & & 0.04 & & 0.01 \\
\hline
\end{tabular}

Data are presented as mean $\pm \mathrm{SD}$; NS: not statistically significant; ${ }^{\star} p<.05 ;{ }^{* *} d \geq .50 ;{ }^{* *} d \geq .80$ 
Next two tables (Table 3, Table 4) answered to RQ2: How will the length of breath holding at rest and after physical load change after a 3-month intervention? These results present the values in the length of breath holding between pre-test and post-test in successfully hit targets at rest and in race load. As in the previous case, no statistically significant dependence was found among most of the monitored results. In the case of standing shooting between the pre-test and post-test in women was statistical significance found $(p<0.05)$, the average length of breath holding $(0.42 \pm 0.36)$ in the pre-test in women's standing shooting is one of the shortest monitored indicators ever. Therefore, it is positive that there was an increase in the duration of apnea after a 3-month intervention period. Cohen's $d$ calculation showed a medium effect size in this case again $(d=0.54)$, just as in other cases were values of Cohen's $d$ trivial or small.

Table 3. Comparison of breath holding (second) at REST (pre-test; hit) vs. at REST (post-test; hit)

\begin{tabular}{l|cc|cc:cc:cc}
\hline & Women_P & $\mathrm{n}_{\mathrm{s}}$ & Men_P & $\mathrm{n}_{\mathrm{s}}$ & Women_S & $\mathrm{n}_{\mathrm{s}}$ & Men_S & $\mathrm{n}_{\mathrm{s}}$ \\
\hline REST_pre-test_hit & $0.53 \pm 0.32$ & 44 & $0.58 \pm 0.36$ & 44 & $0.42 \pm 0.36$ & 42 & $0.61 \pm 0.43$ & 44 \\
REST_post-test_hit & $0.62 \pm 0.31$ & 45 & $0.72 \pm 0.58$ & 46 & $0.66 \pm 0.50$ & 41 & $0.57 \pm 0.46$ & 39 \\
p-value & $\mathrm{NS}(.164)$ & & $\mathrm{NS}(.153)$ & & ${ }^{*} .018$ & & $\mathrm{NS}(.676)$ \\
Cohen's $\boldsymbol{d}$ & 0.31 & & 0.31 & & ${ }^{* *} 0.54$ & & 0.09 \\
\hline
\end{tabular}

Data are presented as mean \pm SD; NS: not statistically significant; ${ }^{*} p<.05 ;{ }^{* *} d \geq .50 ;{ }^{* *} d \geq .80$

Table 4. Comparison of breath holding (second) in RL (pre-test; hit) vs. in RL (post-test; hit)

\begin{tabular}{l|cc:cc:cc:cc}
\hline & Women_P & $\mathrm{n}_{\mathrm{s}}$ & Men_P & $\mathrm{n}_{\mathrm{s}}$ & Women_S & $\mathrm{n}_{\mathrm{s}}$ & Men_S & $\mathrm{n}_{\mathrm{s}}$ \\
\hline RL_pre-test_hit & $0.61 \pm 0.36$ & 68 & $0.75 \pm 0.63$ & 78 & $0.63 \pm 0.43$ & 75 & $0.62 \pm 0.57$ & 81 \\
RL_post-test_hit & $0.69 \pm 0.37$ & 68 & $0.59 \pm 0.46$ & 90 & $0.64 \pm 0.45$ & 70 & $0.57 \pm 0.35$ & 77 \\
$\boldsymbol{p}$-value & $\mathrm{NS}(.247)$ & & $\mathrm{NS}(.055)$ & & $\mathrm{NS}(.925)$ & & $\mathrm{NS}(.516)$ \\
Cohen's $\boldsymbol{d}$ & 0.20 & & 0.30 & & 0.02 & & 0.20 \\
\hline
\end{tabular}

Data are presented as mean \pm SD; NS: not statistically significant; ${ }^{\star} p<.05 ;{ }^{\star \star} d \geq .50 ;{ }^{\star * \star} d \geq .80$

Last two tables (Table 5, Table 6) are related to RQ3. What is the difference in the length of breath holding between hit and missed target? The tables illustrate the length of breath holding between hit and missed targets compared pre-test and post-test at rest and in race load. The results of statistical significance $(p<0.05)$ and large effect size $(d=1.04 ; d=0.89)$ in Table 5 may be distorted by the low number of missed targets. Therefore, let's take the results of the post-test of women and men in the prone position in missed targets with a certain detachment. On the other hand, in the case of women goes on a long-held shots. On the contrary, men fired shots prematurely. Cohen's $d$ points out to large effect size in women and men breath holding in the prone position $(d=1.04 ; d=0.89)$, which is related to the significantly different length of apnea. In the case of statistical significance $(p<0.05)$ in men standing shooting position, it was a longheld shots in average, but it is necessary to emphasize the size of the standard deviation, which indicates both long-held and prematurely fired shots. Cohen's $d$ calculation showed a medium effect size in women and men standing position $(d=0.53 ; d=0.61)$, indicating relatively large differences in the length of apnea. 
Table 5. Comparison of breath holding (second) at REST (hit; pre-test, post-test) vs. at REST (miss; pre-test, post-test)

\begin{tabular}{|c|c|c|c|c|c|c|c|c|}
\hline & Women_P & $\mathrm{n}_{\mathrm{s}}$ & Men_P & $\mathrm{n}_{\mathrm{s}}$ & Women_S & $\mathrm{n}_{\mathrm{s}}$ & Men_S & $\mathrm{n}_{\mathrm{s}}$ \\
\hline REST_pre-test_hit & $0.53 \pm 0.32$ & 44 & $0.58 \pm 0.36$ & 44 & $0.42 \pm 0.36$ & 42 & $0.61 \pm 0.43$ & 44 \\
\hline REST_pre-test_miss & $0.74 \pm 0.67$ & 6 & $0.67 \pm 0.58$ & 11 & $0.66 \pm 0.55$ & 8 & $1.05 \pm 0.90$ & 11 \\
\hline$p$-value & NS (.192) & & NS (.495) & & NS (.115) & & ${ }^{\star} .023$ & \\
\hline Cohen's $d$ & 0.41 & & 0.20 & & ${ }^{* *} 0.53$ & & ${ }^{* *} 0.61$ & \\
\hline REST_post-test_hit & $0.62 \pm 0.31$ & 45 & $0.72 \pm 0.56$ & 50 & $0.66 \pm 0.50$ & 41 & $0.57 \pm 0.46$ & 43 \\
\hline REST_post-test_miss & $1.07 \pm 0.52$ & 5 & $0.25 \pm 0.16$ & 5 & $0.68 \pm 0.58$ & 9 & $0.49 \pm 0.40$ & 12 \\
\hline$p$-value & ${ }^{\star} .008$ & & NS (.102) & & NS (.890) & & NS (.571) & \\
\hline Cohen's $d$ & $* * \star 1.04$ & & $* * * 0.89$ & & 0.05 & & 0.20 & \\
\hline
\end{tabular}

Data are presented as mean \pm SD; NS: not statistically significant; ${ }^{*} p<.05 ;{ }^{* \star} d \geq .50 ;{ }^{* *} d \geq .80$

Table 6 indicates one statistical significance $(p<0.05)$ in men standing shooting in the pretest. It shows, that it may not be appropriate to hold breath for more than 0.6 seconds on average in this age category. All of Cohen's $d$ calculation showed a trivial or small effect size, including the above-mentioned standing shooting in men.

Table 6. Comparison of breath holding (second) in RL (hit; pre-test, post-test) vs. in RL (miss; pre-test, post-test)

\begin{tabular}{|c|c|c|c|c|c|c|c|c|}
\hline & Women_P & $\mathrm{n}_{\mathrm{s}}$ & Men_P & $\mathrm{n}_{\mathrm{s}}$ & Women_S & $\mathrm{n}_{\mathrm{s}}$ & Men_S & $\mathrm{n}_{\mathrm{s}}$ \\
\hline RL_pre-test_hit & $0.61 \pm 0.36$ & 68 & $0.75 \pm 0.63$ & 78 & $0.63 \pm 0.43$ & 75 & $0.62 \pm 0.57$ & 81 \\
\hline $\mathrm{RL}$ _pre-test_miss & $0.49 \pm 0.30$ & 32 & $0.56 \pm 0.73$ & 32 & $0.52 \pm 0.47$ & 25 & $0.89 \pm 0.70$ & 29 \\
\hline$p$-value & NS $(.083)$ & & NS (.183) & & NS (.287) & & ${ }^{*} .049$ & \\
\hline Cohen's $d$ & 0.39 & & 0.27 & & 0.24 & & 0.41 & \\
\hline RL_post-test_hit & $0.69 \pm 0.37$ & 76 & $0.59 \pm 0.46$ & 90 & $0.64 \pm 0.45$ & 78 & $0.57 \pm 0.35$ & 77 \\
\hline RL_post-test_miss & $0.60 \pm 0.41$ & 24 & $0.58 \pm 0.44$ & 20 & $0.83 \pm 0.62$ & 22 & $0.58 \pm 0.40$ & 33 \\
\hline$p$-value & NS (.330) & & NS (.939) & & NS (.132) & & NS $(.847)$ & \\
\hline Cohen's $d$ & 0.23 & & 0.02 & & 0.35 & & 0.04 & \\
\hline
\end{tabular}

Data are presented as mean \pm SD; NS: not statistically significant; ${ }^{*} p<.05 ;{ }^{* \star} d \geq .50 ;{ }^{* *} d \geq .80$

\section{DISCUSSION}

In general, based on the results, it can be stated that to achieve the best possible shooting success, it is appropriate to hold breath for an average of $0.55-0.7$ seconds in both shooting positions for both men and women in this age category.

Focusing on successfully hitting the targets, the length of breath holding should ideally be similar at rest and after exercise, in both the prone and the standing position. We came to this conclusion after analyzing the results of the testing in pre-test and in post-test (Table 1, Table 2). The only statistically significant difference appeared in the women's group in pre-test in the standing position (Table 1). The lower volume of training at the higher load intensity that was 
typical for this training period is suspected to be the reason. In short, women were not able to hold individual shots longer due to poorer position stability at higher HR and RR.

There were no significant differences in the breath holding and the success of shooting between pre-test and post-test (Table 3, Table 4). It was expected that the length of breath holding in October would be longer than in July because of a period of intensive shooting training between the two measurement cycles. However, the relatively short duration of the period between the tests proved not to be significant. A more substantial change is presumed to occur in the next few years when the biathletes should gain greater postural stability, as showed by the studies of Sattlecker et al. $(2014,2017)$. The only statistically significant difference in the shooting success between the time periods was found in women in the standing position (Table 3 ). The supposed reason is a lower postural stability combined with racing load as explained above.

According to the aforementioned results, it cannot be concluded that the length of breath holding has a significant effect on shooting success in physical load, although in some cases a statistical significance has been demonstrated (Table 6). Based on studies by several authors (Coote, 2010; Higginson, 2002; Konttinen et al., 2003; Laaksonen et al., 2018; Pelin et al., 2019; Sattlecker et al., 2014, 2017) it is known that shooting success involves many more factors, such as $\mathrm{HR}$ and RR, postural stability, rifle stability and triggering.

In most cases of shooting at rest and in race load, the result between the hit and miss has been statistically insignificant (Table 5, Table 6), but they showed large effect size in two cases, as described above in the results. In men's standing position, a statistically significant difference was found in shooting at rest and after physical load in pre-test. Similarly to the case of women, this may be associated with the training period, lower position stability, and higher HR and RR in association to Coote (2010). Due to previously described reasons, a portion of the missed targets may have been caused by various other factors. As mentioned in the introduction to the results, if the biathlete is well trained, the apnea pause should be as long at rest and after physical load.

Our research team is currently conducting a study of breath parameters of elite adult biathletes racing in IBU World Cup based on video analysis. The preliminary results show that the length of breath holding is significantly longer in comparison to youth biathletes' performance. The average difference is up to 1 second in both shooting positions. However, the final conclusions of this study are yet to be published.

\section{CONCLUSION}

The aim of the study was to analyze and determine the average length of breath holding in young biathletes and its relation to the success of shooting during 3-months intervention training period. We obtained the necessary data using the Go Direct ${ }^{\oplus}$ Respiration Belt in conjunction with Go Direct ${ }^{\oplus}$ Acceleration Sensor. The comparison of the acquired information with the shooting methodology taught by the coaches brought beneficial results. The length of breath holding corresponded to the recommendations of the coaches in the Czech Republic - namely of 0.5 to 1 second in the prone and 0.2 to 1 second in the standing position. We can clarify this statement, as the study shows that is appropriate to hold breath for an average of $0.55-0.7$ seconds in both shooting positions for both men and women in this age category.

The findings should help biathlon coaches and the biathletes themselves. Breathing technique is one of the basics of shooting performance, so it should be widely represented in the shooting training of young athletes. With increasing postural and rifle stability, the length of breath holding should keep extending over several years. At the transition from the junior to the senior category, 
the biathlete should be able to hold their breath before the shot for approximately 1 second in both prone and standing position. However, this is an individual matter as stated before.

Finally, it is necessary to mention the limiting factors of our study. We consider the schedule of the measurements to be one of the major ones. The research had to be conducted in summer and autumn in concord with the schedule of the Specific University Research Grant. For comparison, it would be beneficial to add data from the following winter. Furthermore, we did not report the results of the length of breath holding of elite biathletes. As mentioned above, this is the subject of a different study.

Based on the results, we affirm that an adequate length of breath holding should precede each shot. As indicated by the results of the standard deviation of missed targets, very short or unnecessary long breath holding can lead to worse shooting performance.

\section{ACKNOWLEDGEMENTS}

This article was written at the Masaryk University as a part of the project "Evaluation of shooting performance based on selected aspects of physical load II." number MUNI/A/1271/2019 with the support of the Specific University Research Grant, as provided by the Ministry of Education, Youth and Sports of the Czech Republic in the year 2020.

\section{REFERENCES}

Cohen, J. (1988). Statistical power analysis for the behavioural sciences (2. Ed.). Hillsdale, NJ: Erlbaum.

Coote, J. H. (2010). Recovery of heart rate following intense dynamic exercise. Experimental physiology, 95(3), 431-440. https://doi.org/10.1113/expphysiol.2009.047548

Higginson, B. K. (2002). Effect of exercise intensity on shooting performance in the sport of summer biathlon. Doctoral dissertation. Montana State University-Bozeman, College of Education, Health \& Human Development.

Høydal, K. L., \& Nord, I. (2017). The importance of heart rate monitors in controlling intensity during training and competition in junior biathlon athletes. Journal of Human Sport and Exercise, 12(2), 358-366. https://doi.org/10.14198/ jhse.2017.122.12

Ihalainen, S., Laaksonen, M. S., Kuitunen, S., Leppävuori, A., Mikkola, J., Lindinger, S. J., \& Linnamo, V. (2018). Technical determinants of biathlon standing shooting performance before and after race simulation. Scandinavian journal of medicine \& science in sports, 28(6), 1700-1707. https://doi.org/10.1111/sms.13072

Konttinen, N., Mets, T., Lyytinen, H., \& Paananen, M. (2003). Timing of triggering in relation to the cardiac cycle in nonelite rifle shooters. Research quarterly for exercise and sport, 74(4), 395-400. http://dx.doi.org/10.1136/bjsm.21.1.33

Laaksonen, M. S., Finkenzeller, T., Holmberg, H. C., \& Sattlecker, G. (2018). The influence of physiobiomechanical parameters, technical aspects of shooting, and psychophysiological factors on biathlon performance: A review. Journal of sport and health science, 7(4), 394-404. https://doi.org/10.1016/j.jshs.2018.09.003

Pelin, B. I., \& Mereuţă, C. (2018). Improvement of shooting technical skills in the shooting range within the biathlon test for juniors. Bulletin of the Transilvania University of Brasov. Series IX, Sciences of Human Kinetics, 11(2), 179-184.

Pelin, B. I., Bondoc-lonescu, D., \& Turcu, I. (2019). Optimising technical shooting skills at the shooting range by adjusting the intensity of the effort to junior biathletes. Discobolul - Physical Education, Sport and Kinetotherapy Journal, 58(4), 32-36.

Pelin, B., \& Bondoc-lonescu, D. (2020). Shooting range improvement by monitoring the discriminating factors of the junior biathletes. Bulletin of the Transilvania University of Brasov, Series IX: Sciences of Human Kinetics, 13(2), 61-68. https://doi. org/10.31926/but.shk.2020.13.62.2.7

Sattlecker, G., Buchecker, M., Müller, E., \& Lindinger, S. J. (2014). Postural balance and rifle stability during standing shooting on an indoor gun range without physical stress in different groups of biathletes. International Journal of Sports Science \& Coaching, 9(1), 171-184.

Sattlecker, G., Buchecker, M., Gressenbauer, C., Müller, E., \& Lindinger, S. J. (2017). Factors discriminating high from low score performance in biathlon shooting. International journal of sports physiology and performance, 12(3), 377-384. http://dx.doi.org/10.1123/ijspp.2016-0195

Vonheim, A. (2012). The effect of skiing intensity on shooting performance in biathlon. Master thesis. Trondheim, Norwegian University of Science and Technology.

Žák, M., Struhár, I., Janoušek, D., \& Ondráček, J. (2020). Dynamic rifle stability is not influenced by exercise intensity in young biathletes. Journal of Human Sport and Exercise, 15(4), 762-771. https://doi.org/10.14198/jhse.2020.154.04

\section{Contact Information}

Mgr. Michal Žák, email: 392472@mail.muni.cz; Department of Athletics, Swimming and Outdoor Sports. 